\title{
Measurement of Power Density in a Lossy Material by means of Electromagnetically induced acoustic signals for non-invasive determination of spatial thermal absorption in connection with pulsed hyperthermia
}

\author{
F.Caspers* and J.Conway**
}

\begin{abstract}
For non-invasive determination of the spatial power density distribution during RF- and microwave hyperthermia it is proposed to apply the electromagnetic energy as short, high intensity pulses. This pulsed signal should have the same average power and thus give the same temperature elevation as the CW source usually applied. Due to the high peak power of the equivalent pulsed signal, with a duty cycle $<1: 100$, externally measurable thermoacoustic oscillations are induced in the irradiated object. They can be evaluated for the reconstruction of a spatial power density profile.

* Institut fur Hoch- und Hochstfrequenztechnik der Ruhr-Universitat Bochum, West Germany. (With CERN, Geneva, Switzerland since 17.5.82.)

** Sheffield University and Area Health Authority, Western Park Hospital,Sheffield, UK.
\end{abstract}

